ARTICLE

Received 9 Dec 2016 | Accepted 27 Jan 2017 | Published 24 Mar $2017 \quad$ DOl: 10.1038/ncomms14739

\title{
The fourth crystallographic closest packing unveiled in the gold nanocluster crystal
}

Zibao Gan ${ }^{1, \star}$, Jishi Chen ${ }^{1, \star}$, Juan Wang ${ }^{2}$, Chengming Wang ${ }^{3}$, Man-Bo Li', Chuanhao Yao ${ }^{1}$, Shengli Zhuang ${ }^{1}$, An $\mathrm{Xu}^{2}$, Lingling $\mathrm{Li}^{4} \&$ Zhikun $\mathrm{Wu}^{1}$

Metal nanoclusters have recently attracted extensive interest not only for fundamental scientific research, but also for practical applications. For fundamental scientific research, it is of major importance to explore the internal structure and crystallographic arrangement. Herein, we synthesize a gold nanocluster whose composition is determined to be $\mathrm{Au}_{60} \mathrm{~S}_{6}\left(\mathrm{SCH}_{2} \mathrm{Ph}\right)_{36}$ by using electrospray ionization mass spectrometry and single crystal X-ray crystallography (SCXC). SCXC also reveals that $\mathrm{Au}_{60} \mathrm{~S}_{6}\left(\mathrm{SCH}_{2} \mathrm{Ph}\right)_{36}$ consists of a fcc-like $\mathrm{Au}_{20}$ kernel protected by a pair of giant $\mathrm{Au}_{20} \mathrm{~S}_{3}\left(\mathrm{SCH}_{2} \mathrm{Ph}\right)_{18}$ staple motifs, which contain 6 tetrahedral-coordinate $\mu_{4}$-S atoms not previously reported in the Au-S interface. Importantly, the fourth crystallographic closest-packed pattern, termed $6 \mathrm{H}$ left-handed helical $(6 \mathrm{HLH})$ arrangement, which results in the distinct loss of solid photoluminescence of amorphous $\mathrm{Au}_{60} \mathrm{~S}_{6}\left(\mathrm{SCH}_{2} \mathrm{Ph}\right)_{36}$, is found in the crystals of $\mathrm{Au}_{60} \mathrm{~S}_{6}\left(\mathrm{SCH}_{2} \mathrm{Ph}\right)_{36}$. The solventpolarity-dependent solution photoluminescence is also demonstrated. Overall, this work provides important insights about the structure, Au-S bonding and solid photoluminescence of gold nanoclusters.

\footnotetext{
${ }^{1}$ Key Laboratory of Materials Physics, Anhui Key Laboratory of Nanomaterials and Nanotechnology, CAS Center for Excellence in Nanoscience, Institute of Solid State Physics, Chinese Academy of Sciences, Hefei 230031, China. ${ }^{2}$ Key Laboratory of lon Beam Bioengineering, Institute of Technical Biology and Agriculture Engineering, Chinese Academy of Sciences, Hefei 230031, China. ${ }^{3}$ Hefei National Laboratory for Physical Sciences at the Microscale, University of Science and Technology of China, Hefei 230026, China. ${ }^{4}$ Instrumental Analysis Center, Shanghai Jiaotong University, Shanghai 200240, China.

* These authors contributed equally to this work. Correspondence and requests for materials should be addressed to Z.W. (email: zkwu@issp.ac.cn).
} 
B ridging gold atom (or gold complexes) ${ }^{1-3}$ and nanocrystals (typically $>3 \mathrm{~nm})^{4-8}$, gold nanoclusters ${ }^{9-15}$ have recently attracted increasing research interest due to their welldefined compositions and structures, unique properties and potential applications. In particular, the structures of gold nanoclusters are of primary significance and have received the most extensive attention in the research community ${ }^{16-21}$. However, structure achievements remain limited due to the difficulty of precise size control and structural resolution, especially for relatively large gold nanoclusters ${ }^{22-26}$. It is generally believed that the gold nanoclusters adopt kernel-staple structures, and the staple motifs play a vital role in stabilizing the nanoclusters. The large staple motif $\mathrm{Au}_{8}(\mathrm{SR})_{8}$ was reported in $\mathrm{Au}_{20}(\mathrm{SR})_{16}$ nanocluster ${ }^{27}$, but the question remains whether there is a larger staple motif. Although thiolate or sulfido (-S-) can be found in the surface of gold nanoclusters as protecting ligands, both thiolate sulfur and sulfido (-S-) are always three-coordinate (mainly $\mu_{2}-\mathrm{S}$ and rarely $\left.\mu_{3}-\mathrm{S}\right)^{28,29}$, naturally raising the question of whether there are sulfurs with any other coordination numbers in gold nanoclusters. Notably, four-coordinate $\mu_{4}$-S and fivecoordinate $\mu_{4}$-S have been reported in $\mathrm{Cr}(\mathrm{III})$ and $\mathrm{Cu}(\mathrm{I})$ complexes $^{30,31}$, respectively, but such high-coordinate sulfur has not been found in $\mathrm{Au}-\mathrm{S}$ interfaces (not limited to gold nanoclusters).

In addition to these internal structure questions, there are some other crystallographic arrangement issues that need to be addressed. It is known that there are two classic crystallographic closest packings for atomic crystals: the fcc arrangement with a packing sequence of 'ABC' found in bulk $\mathrm{Au}, \mathrm{Ag}, \mathrm{Pd}, \mathrm{Pt}$ and $\mathrm{Ir}$ metals $^{32}$, FePt nanoparticles ${ }^{33}$, and others; and the hcp or $2 \mathrm{H}$ arrangement with a packing sequence of ' $A B$ ' revealed in $\mathrm{Rh}$ nanosheets ${ }^{34}$, $\mathrm{Au}$ square sheets ${ }^{35}$, $\mathrm{PbPt}$ nanorods ${ }^{36}$, BiPt nanoplates ${ }^{37}$, among others. The third closest packing, named the $4 \mathrm{H}$ arrangement, has a packing sequence of 'ABCD' and was first observed in bulk Ag by Novgorodova et al. in 1979 (ref. 38), then subsequently revealed in Ag nanocrystals and nanowires ${ }^{39,40}, \mathrm{Au}$ nanoribbons ${ }^{41}$ and more. The advances in gold nanocluster research provide opportunities to discover novel closest-packed patterns. It is worth noting that in contrast to gold nanocrystals, molecule-like gold nanoclusters can grow high-quality single crystals for X-ray crystallography analyses. Thus, not only the internal structure (atom packing in every gold nanocluster) but also the crystallographic arrangement (the arrangement of gold nanoclusters in single crystals) of gold nanoclusters can be resolved by single crystal $\mathrm{X}$-ray crystallography (SCXC). Jin et al. reported the fcc crystallographic arrangement of $\mathrm{Au}_{30}(\mathrm{SR})_{18}$ nanoclusters in early 2016 (ref. 42), and very recently, $\mathrm{Wu}$ et al. reported the $4 \mathrm{H}$ crystallographic arrangement in $\mathrm{Au}_{92}$ crystals $^{43}$. It is currently unknown whether there are any other intriguing arrangement patterns in gold nanoclusters. To address this question, together with the above mentioned issues, more gold nanoclusters with complex surfaces must be synthesized, and their structures must be resolved.

Herein, we report the synthesis, structure (including internal and crystallographic) and photoluminescence of a gold nanocluster whose composition is determined to be $\mathrm{Au}_{60} \mathrm{~S}_{6}\left(\mathrm{SCH}_{2} \mathrm{Ph}\right)_{36}$ using electrospray ionization mass spectrometry (ESI-MS) and SCXC. In particular, we find a fourth closest packing in the crystal of $\mathrm{Au}_{60} \mathrm{~S}_{6}\left(\mathrm{SCH}_{2} \mathrm{Ph}\right)_{36}$.

\section{Results}

Synthesis and characterization. The $\mathrm{Au}_{60} \mathrm{~S}_{6}\left(\mathrm{SCH}_{2} \mathrm{Ph}\right)_{36}$ nanocluster was obtained via a thermal-induced ligand exchange reaction of molecularly pure $\mathrm{Au}_{38}\left(\mathrm{SC}_{2} \mathrm{H}_{4} \mathrm{Ph}\right)_{24}$ with excess phenylmethanethiol $\left(\mathrm{HSCH}_{2} \mathrm{Ph}\right)$. Of note, the interesting thing in this synthesis is that the protecting ligand of the starting nanoclusters and the incoming ligand only have subtle difference in composition $\left(-\mathrm{CH}_{2}-\right.$, see Supplementary Fig. 1), which may have some implications to other nanoparticles (including quantum dots) synthesis. Briefly, the reaction was initialized by dissolving $10 \mathrm{mg}$ of $\mathrm{Au}_{38}\left(\mathrm{SC}_{2} \mathrm{H}_{4} \mathrm{Ph}\right)_{24}$ nanoclusters in $1 \mathrm{ml}$ of toluene containing $0.5 \mathrm{ml}$ of $\mathrm{HSCH}_{2} \mathrm{Ph}$, with stirring. After proceeding overnight at $100^{\circ} \mathrm{C}$ under nitrogen atmosphere, the reaction was terminated by the addition of plenty of methanol. The crude product was thoroughly washed with petroleum ether and methanol for four times, then subjected to subsequent separation and purification by preparative thin-layer chromatography (PTLC $)^{19}$. Single crystals of the purified nanoclusters were grown by the vapour diffusion of acetonitrile into the toluene solution of the purified nanoclusters at $5^{\circ} \mathrm{C}$. Black-coloured crystals were formed after one week. As shown in Fig. 1a, the optical absorption spectrum of the as-obtained nanocluster has no dominant visible absorption peak and only shows a very weak absorption at $\sim 345 \mathrm{~nm}(3.59 \mathrm{eV})$ and a step at $\sim 600 \mathrm{~nm}$ $(2.07 \mathrm{eV})$. The optical energy gap was determined to be $\sim 1.73 \mathrm{eV}$ by extrapolating the lowest-energy absorption peak to zero absorbance (see the inset in Fig. 1a). The composition of the as-obtained nanocluster was identified by ESI-MS. Of note, without the addition of cesium acetate ( $\mathrm{CsOAc}$ ), no signal was observed in either positive or negative mode, which implies the charge neutrality of the nanocluster. To impart charges, CsOAc was added to the nanocluster solution to form positively charged
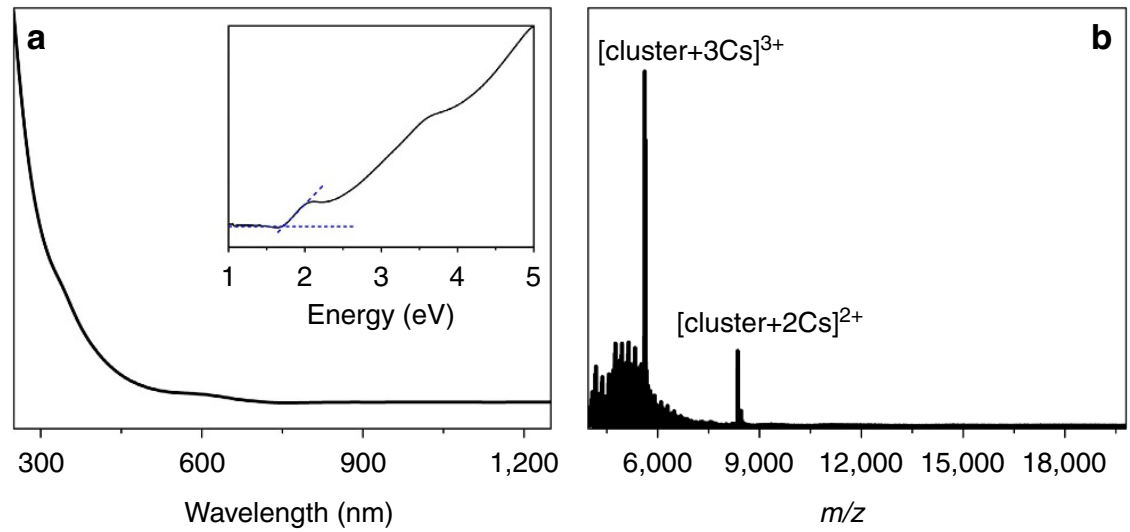

Figure 1 | Characterization of $\mathbf{A u}_{\mathbf{6 0}} \mathbf{S}_{\mathbf{6}}\left(\mathbf{S C H}_{\mathbf{2}} \mathbf{P h}\right)_{\mathbf{3 6}}$. Ultraviolet-visible-near-infrared spectrum (a) and electrospray ionization mass spectrometry (ESI-MS) (positive ion mode) spectrum (b) of $\mathrm{Au}_{60} \mathrm{~S}_{6}\left(\mathrm{SCH}_{2} \mathrm{Ph}\right)_{36}$. The inset of a represents the spectrum on the energy scale. 


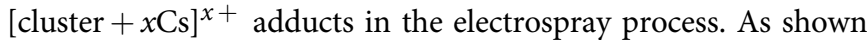
in Fig. 1b, two intense peaks at mass/charge ratio $(\mathrm{m} / \mathrm{z})$ 8,356.22 and 5,615.49 are observed, which can be readily assigned to [ $\left.\mathrm{Au}_{60} \mathrm{~S}_{6}\left(\mathrm{SCH}_{2} \mathrm{Ph}\right)_{36} \mathrm{Cs}_{2}\right]^{2+}$ (calculated: 8,356.31, deviation: 0.09) and $\left[\mathrm{Au}_{60} \mathrm{~S}_{6}\left(\mathrm{SCH}_{2} \mathrm{Ph}\right)_{36} \mathrm{Cs}_{3}\right]^{3+}$ (calculated: 5,615.51, deviation: $0.02)$, respectively. Thus, the as-obtained nanocluster should be $\mathrm{Au}_{60} \mathrm{~S}_{6}\left(\mathrm{SCH}_{2} \mathrm{Ph}\right)_{36}$, which was further confirmed by the subsequent SCXC analysis.

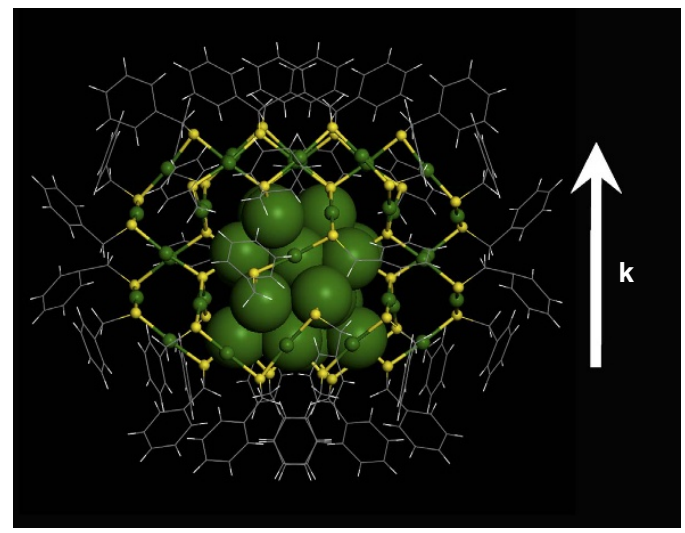

Figure 2 | X-ray atomic structure of the $\mathrm{Au}_{60} \mathrm{~S}_{6}\left(\mathbf{S C H}_{2} \mathbf{P h}\right)_{36}$ nanocluster. The $\mathrm{Au}$ atoms are in green, the sulfur atoms are in yellow, and the benzyls are in wireframe. The vertical upward arrow indicates the direction of the k-vector.
Internal structure. The structure of the as-obtained nanocluster was determined by SCXC. Figure 2 presents the total structure of the as-obtained nanocluster, which crystallizes in a hexagonal P6522 space group, has no centre or plane of symmetry and only possesses a $C_{2}$ rotation axis of symmetry. The as-obtained nanocluster consists of an $\mathrm{Au}_{20}$ kernel and a pair of giant $\mathrm{Au}_{20} \mathrm{~S}_{3}\left(\mathrm{SCH}_{2} \mathrm{Ph}\right)_{18}$ staple motifs. The $\mathrm{Au}_{20}$ kernel can be viewed as a fragment of the fcc structure in bulk gold or nanoparticles, typically $>3 \mathrm{~nm}$ (diameter). The 16 gold atoms in the kernel constitute an $\mathrm{Au}_{16}$ tetrahedron without vertex (Fig. 3a,e), and each facet of the tetrahedron is a distorted hexagon. The other 4 $\mathrm{Au}$ atoms (highlighted in blue and red, Fig. 3b,f) in the kernel are capped on the four facets of the tetrahedron in a one-to-one fashion. Moreover, the $\mathrm{Au}_{20}$ kernel is protected by a pair of giant unanimous $\mathrm{Au}_{20} \mathrm{~S}_{3}\left(\mathrm{SCH}_{2} \mathrm{Ph}\right)_{18}$ staple motifs: one $\mathrm{Au}_{20} \mathrm{~S}_{3}\left(\mathrm{SCH}_{2} \mathrm{Ph}\right)_{18}$ staple connects to the $\mathrm{Au}_{20}$ kernel (Fig. 3c,g; the back view can be found in Supplementary Fig. 2) by five terminal $\mu_{2}$-S atoms (binding to one kernel Au atom, one staple $\mathrm{Au}$ atom and one $-\mathrm{CH}_{2} \mathrm{Ph}$ group, indicated by white arrow, see Fig. 3i,j) and three bridging $\mu_{4}-\mathrm{S}$ atoms (binding to one kernel $\mathrm{Au}$ atom and three staple $\mathrm{Au}$ atoms, highlighted in the white circle, see Fig. 3i,j), and the other $\mathrm{Au}_{20} \mathrm{~S}_{3}\left(\mathrm{SCH}_{2} \mathrm{Ph}\right)_{18}$ staple binds to the $\mathrm{Au}_{20}$ kernel in the same fashion after rotating $\left(180^{\circ}\right)$ along the $\mathrm{C}_{2}$ axis of symmetry (Fig. 3d,h). It is worth noting that in addition to the common three-coordinate $\mu_{2}-\mathrm{S}$ atoms (binding to one $\mathrm{R}$ group and two $\mathrm{Au}$ atoms ( $\mathrm{Au}-\mathrm{SR}-\mathrm{Au})$, see Fig. $3 \mathrm{k})$, there are six tetrahedral-coordinate $S$ atoms $\left(\mu_{4}-\mathrm{S}\right)$ (every $\mu_{4}$-S binds to four $\mathrm{Au}$ atoms, see Fig. 31), indicating the uniqueness of $\mathrm{Au}-\mathrm{S}$ bonding in the $\mathrm{Au}_{60} \mathrm{~S}_{6}\left(\mathrm{SCH}_{2} \mathrm{Ph}\right)_{36}$ nanocluster. Herein, the six surprising $\mu_{4}$-S atoms should come from the thiol, which may undergo S-C bond cleavage under heating conditions during the ligand-

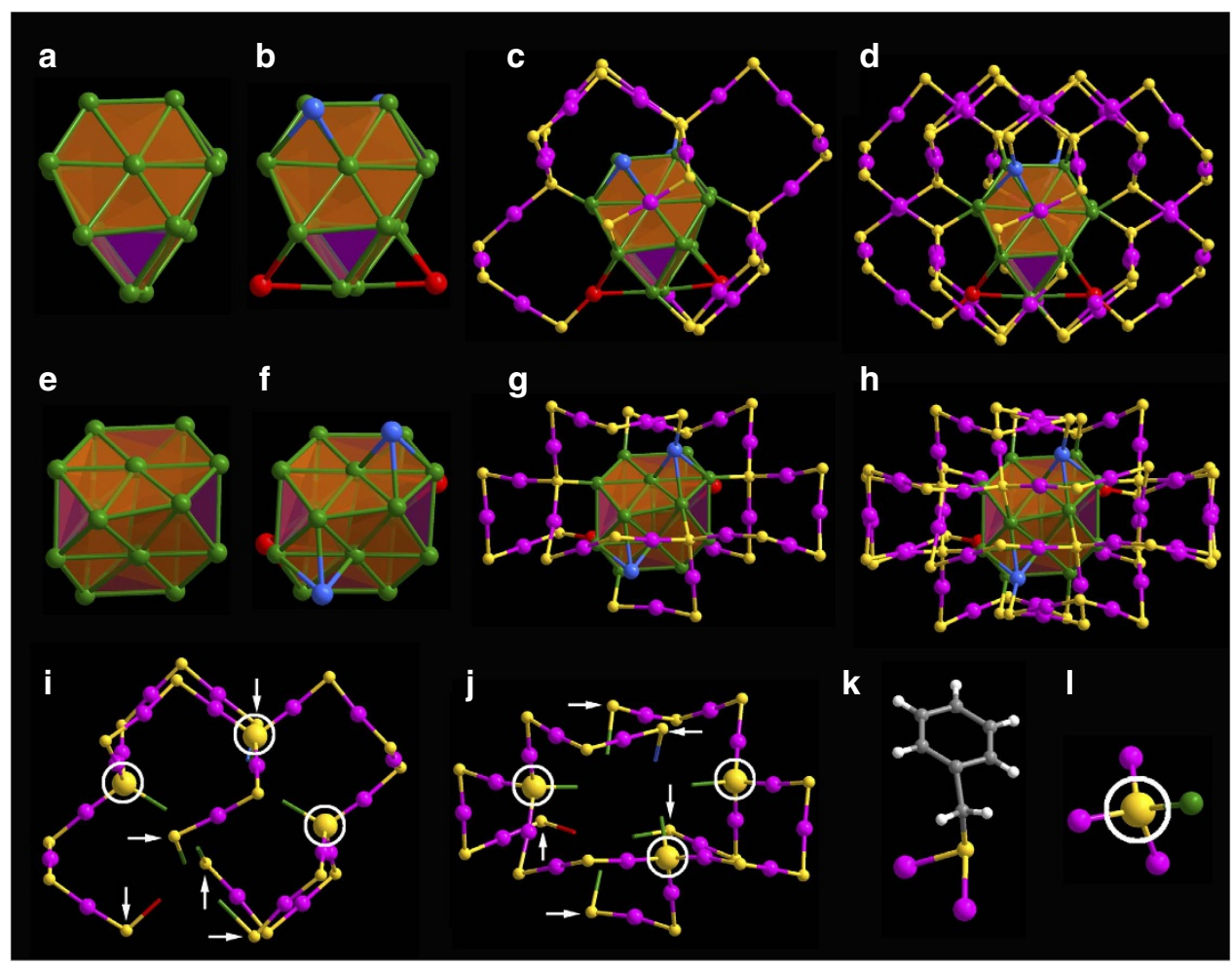

Figure 3 | Anatomy of the atomic structure of $\mathbf{A u}_{\mathbf{6 0}} \mathbf{S}_{\mathbf{6}}\left(\mathbf{S C H}_{\mathbf{2}} \mathbf{P h}\right)_{\mathbf{3}}$ nanocluster. (a,e) Side and top view of the $A u_{16}$ tetrahedron without vertex; $(\mathbf{b}, \mathbf{f})$ side and top view of $\mathrm{Au}_{20}$ inner kernel; $(\mathbf{c}, \mathbf{g})$ side and top view of $\mathrm{Au}_{20}$ kernel with an $\mathrm{Au}_{20} \mathrm{~S}_{3}\left(\mathrm{SCH}_{2} \mathrm{Ph}\right)_{18}$ staple motif; (d, $\left.\mathbf{h}\right)$ side and top view of the overall framework of the $\mathrm{Au}_{60} \mathrm{~S}_{6}\left(\mathrm{SCH}_{2} \mathrm{Ph}\right)_{36}$ nanocluster with a pair of $\mathrm{Au}_{20} \mathrm{~S}_{3}\left(\mathrm{SCH}_{2} \mathrm{Ph}\right)_{18}$ staple motifs; $(\mathbf{i}, \mathbf{j})$ side and top view of the $\mathrm{Au}_{20} \mathrm{~S}_{3}\left(\mathrm{SCH}_{2} \mathrm{Ph}\right)_{18}$ staple motif; (k) the $\mu_{2}-\mathrm{S}$ atom and (I) the $\mu_{4}-\mathrm{S}$ atom (highlighted in the white circle). Colour labels: yellow $=\mathrm{S}$, white $=\mathrm{H}$, gray $=\mathrm{C}$, other colour $=\mathrm{Au}$. The Au atoms are represented in different colours to differentiate from them each other on the bonding ways and occupying positions. 


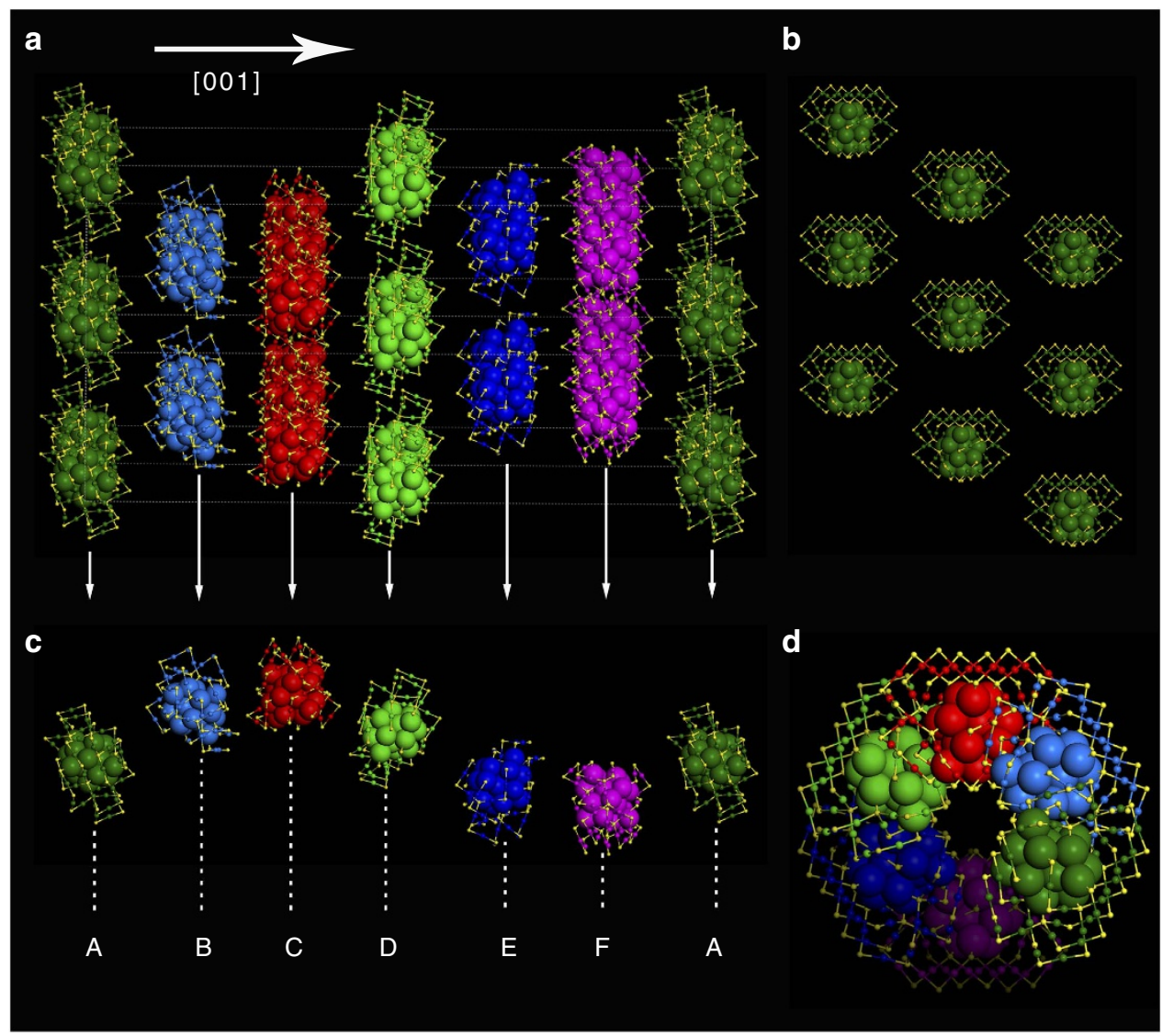

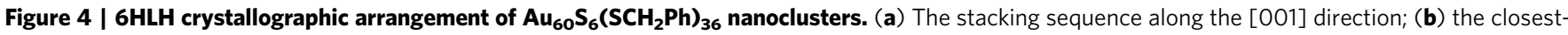
packed pattern of $\mathrm{Au}_{60} \mathrm{~S}_{6}\left(\mathrm{SCH}_{2} \mathrm{Ph}\right)_{36}$ nanoclusters in the (001) plane; $(\mathbf{c}, \mathbf{d})$ different views of the left-handed helical arrangement of $\mathrm{Au}_{60} \mathrm{~S}_{6}\left(\mathrm{SCH}_{2} \mathrm{Ph}_{36}\right.$ nanoclusters. (Note: to highlight the $6 \mathrm{HLH}$ arrangement, the Au atoms of the nanoclusters in each close-packed plane are labeled in different colours).

exchange-induced structure transformation process ${ }^{42}$. A single $\mathrm{Au}_{20} \mathrm{~S}_{3}(\mathrm{SR})_{18}$ staple motif (branched at the site of the $\mu_{4}-\mathrm{S}$ atom) contains two -Au-SR-Au- and three -Au-SR-Au-SR-Au- units between the five terminal $\mu_{2}-\mathrm{S}$ atoms and three bridging $\mu_{4}-\mathrm{S}$ atoms, and one -Au-SR-Au-SR-Au- and one -Au-SR-Au-SR-AuSR-Au- units among the three bridging $\mu_{4}$-S atoms (Fig. $3 j$ ). Of note, such a giant staple motif has not been found in thiolated gold nanoclusters, which is larger than the $\mathrm{Au}_{8}(\mathrm{SR})_{8}$ staple motif in $\mathrm{Au}_{20}(\mathrm{SR})_{16}$ nanoclusters ${ }^{27}$.

Crystallographic arrangement. Interestingly, $\mathrm{Au}_{60} \mathrm{~S}_{6}\left(\mathrm{SCH}_{2} \mathrm{Ph}\right)_{36}$ nanoclusters adopt the closest packing, and a very special stacking sequence of 'ABCDEF' along the close-packed [001] direction is found in its single crystals (Fig. 4a). The $\mathrm{Au}_{60} \mathrm{~S}_{6}\left(\mathrm{SCH}_{2} \mathrm{Ph}\right)_{36}$ nanoclusters in every stacking layer $((001)$ plane) are arranged uniformly, and each nanocluster is surrounded by six identical nanoclusters with the same tropism (k-vector), as shown in Fig. $4 \mathrm{~b}$ and Supplementary Fig. 3. Moreover, the stacking layer perpendicular to the [001] direction can overlap completely with its neighboring layer after every nanocluster in the layer rotates $\left(60^{\circ}\right)$ clockwise or anti-clockwise along the $z$ axis (Supplementary Fig. 3), and thus the arrangement of $\mathrm{Au}_{60} \mathrm{~S}_{6}\left(\mathrm{SCH}_{2} \mathrm{Ph}\right)_{36}$ nanoclusters in single crystals along the [001] direction is reminiscent of the left-handed helix (Supplementary Fig. 4). For clarity, a left-handed helical sequence, here termed the $6 \mathrm{H}$ left-handed helical (6HLH) arrangement, is isolated from the crystal, as shown in Fig. 4c,d. Such a crystallographic arrangement is not only interesting but also exciting, as the third closest packing in crystals, named $4 \mathrm{H}$, was found in 1979 (ref. 38).

Photoluminescence. The $6 \mathrm{HLH}$ arrangement indicates the unique interactions among $\mathrm{Au}_{60} \mathrm{~S}_{6}\left(\mathrm{SCH}_{2} \mathrm{Ph}\right)_{36}$ nanoclusters in the single crystals, which is supported by the photoluminescence intensity comparison between the amorphous and crystallized $\mathrm{Au}_{60} \mathrm{~S}_{6}\left(\mathrm{SCH}_{2} \mathrm{Ph}\right)_{36}$ as shown in Fig. 5. Although the emission spectrum profiles of the disordered (amorphous) $\mathrm{Au}_{60} \mathrm{~S}_{6}\left(\mathrm{SCH}_{2} \mathrm{Ph}\right)_{36}$ is almost superimposable to that of the ordered (crystallized) $\mathrm{Au}_{60} \mathrm{~S}_{6}\left(\mathrm{SCH}_{2} \mathrm{Ph}\right)_{36}$, the emission intensity of the former is $\sim 1.7$ folds of that of the latter. The lower photoluminescence intensity in crystallized $\mathrm{Au}_{60} \mathrm{~S}_{6}\left(\mathrm{SCH}_{2} \mathrm{Ph}\right)_{36}$ might be caused by the energy transfer among the $6 \mathrm{HLH}$ arranged $\mathrm{Au}_{60} \mathrm{~S}_{6}\left(\mathrm{SCH}_{2} \mathrm{Ph}\right)_{36}$ nanoclusters, indicating the notable interactions among the $6 \mathrm{HLH}$ arranged $\mathrm{Au}_{60} \mathrm{~S}_{6}\left(\mathrm{SCH}_{2} \mathrm{Ph}\right)_{36}$ nanoclusters. Although the $6 \mathrm{HLH}$ arrangement barely changes the emission spectrum, solvent does result in the obvious blueshift of the maximum emission peak, and interestingly, the blueshift increases with the increase of solvent polarity, while the photoluminescence intensity of $\mathrm{Au}_{60} \mathrm{~S}_{6}\left(\mathrm{SCH}_{2} \mathrm{Ph}\right)_{36}$ nanoclusters decreases with the increase of solvent polarity (see Fig. 6). Anyway, these facts indicate that the solution photoluminescence of $\mathrm{Au}_{60} \mathrm{~S}_{6}\left(\mathrm{SCH}_{2} \mathrm{Ph}\right)_{36}$ is solvent-polarity dependent.

\section{Discussion}

In summary, a novel near-infrared-emissive gold nanocluster was synthesized via a thermal-induced ligand exchange process, and 


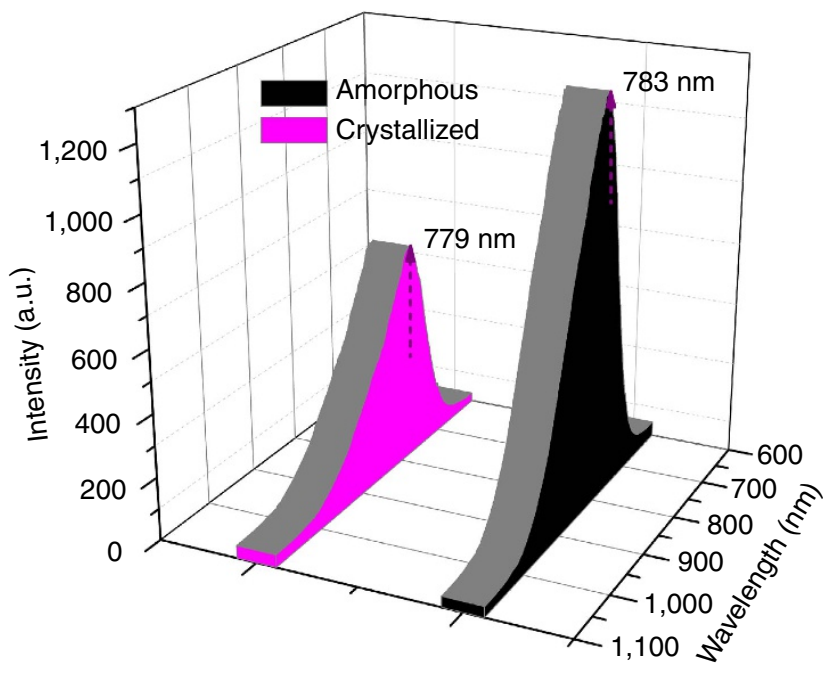

Figure 5 | The solid photoluminescence spectra of $\mathbf{A u}_{60} \mathbf{S}_{6}\left(\mathbf{S C H}_{\mathbf{2}} \mathrm{Ph}\right)_{36}$ nanoclusters. $\mathrm{Au}_{60} \mathrm{~S}_{6}\left(\mathrm{SCH}_{2} \mathrm{Ph}\right)_{36}$ nanoclusters in crystallized and amorphous states exhibit obviously different photoluminescence intensities but almost identical emission spectrum profiles. Note: the excitation wavelength $\lambda_{\mathrm{ex}}=514 \mathrm{~nm}$.

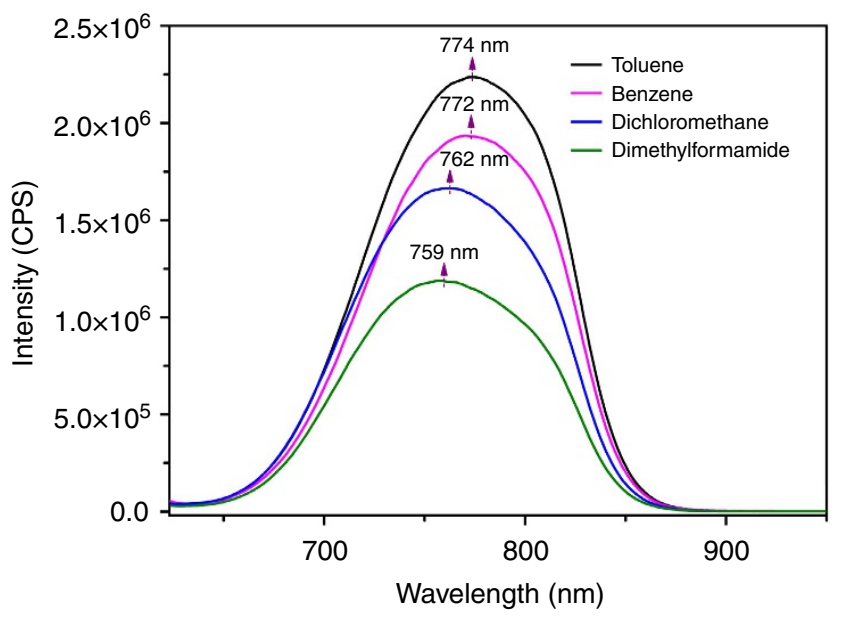

Figure 6 | The solvent-polarity-dependent solution photoluminescence of $\mathbf{A u}_{\mathbf{6 0}} \mathbf{S}_{\mathbf{6}}\left(\mathbf{S C H}_{\mathbf{2}} \mathbf{P h}\right)_{\mathbf{3 6}}$ nanoclusters. Not only the maximum emission wavelengths but also the photoluminescence intensities of $\mathrm{Au}_{60} \mathrm{~S}_{6}\left(\mathrm{SCH}_{2} \mathrm{Ph}\right)_{36}$ nanoclusters in solution are dependent on the solvent polarity. Note: the excitation wavelength $\lambda_{\mathrm{ex}}=514 \mathrm{~nm}$.

its composition was determined to be $\mathrm{Au}_{60} \mathrm{~S}_{6}\left(\mathrm{SCH}_{2} \mathrm{Ph}\right)_{36}$ using ESI-MS and SCXC. SCXC also revealed that the nanocluster consists of one fcc-like $\mathrm{Au}_{20}$ kernel protected by a pair of $\mathrm{Au}_{20} \mathrm{~S}_{3}\left(\mathrm{SCH}_{2} \mathrm{Ph}\right)_{18}$ giant staple motifs, which are larger than the existing staple motifs in structurally resolved gold nanoclusters. In particular, three tetrahedral-coordinate $\mu_{4}$-S atoms were found enclosed in every giant staple motif, which challenges the conventional opinion that sulfur in the $\mathrm{Au}-\mathrm{S}$ interface is always three-coordinate $\mu_{2}$-S. Most important of all, a $6 \mathrm{HLH}$ crystallographic arrangement was found in the crystal of $\mathrm{Au}_{60} \mathrm{~S}_{6}\left(\mathrm{SCH}_{2} \mathrm{Ph}\right)_{36}$ nanoclusters, and this finding also represents an advance in crystallographic packing research since the $4 \mathrm{H}$ phase finding in 1979. Interestingly, the $6 \mathrm{HLH}$ arrangement gives rise to the obvious loss of solid photoluminescence of amorphous $\mathrm{Au}_{60} \mathrm{~S}_{6}\left(\mathrm{SCH}_{2} \mathrm{Ph}\right)_{36}$, indicating the strong interaction among the uniquely arranged nanoclusters. Another interesting finding is the solvent-polarity-dependent solution photoluminescence of
$\mathrm{Au}_{60} \mathrm{~S}_{6}\left(\mathrm{SCH}_{2} \mathrm{Ph}\right)_{36}$ nanoclusters. Briefly, this work provides new and exciting views to the structure, $\mathrm{Au}-\mathrm{S}$ bonding and photoluminescence (including solid state and solution) of gold nanoclusters, and our work is expected to stimulate further research on the structure and properties of crystallized materials at the nanoscale.

\section{Methods}

Reagents. All chemicals are commercially available and used as received. Tetraoctylammonium bromide (TOAB, 98.0\%), 2-Phenylethanethiol $\left(\mathrm{PhC}_{2} \mathrm{H}_{4} \mathrm{SH}\right.$, 99.0\%) and phenylmethanethiol $\left(\mathrm{PhCH}_{2} \mathrm{SH}, 99.0 \%\right)$ were purchased from SigmaAldrich. Tetrachloroauric(III) acid $\left(\mathrm{HAuCl}_{4} \cdot 4 \mathrm{H}_{2} \mathrm{O}, 99.7 \%\right)$, sodium borohydride $\left(\mathrm{NaBH}_{4}, 98.0 \%\right)$, acetonitrile (99.0\%, AR), dichloromethane $\left(\mathrm{CH}_{2} \mathrm{Cl}_{2}, 99.0 \%, \mathrm{AR}\right)$, tetrahydrofuran (THF, $99.0 \%$, AR), toluene (99.5\%, AR), methanol $\left(\mathrm{CH}_{3} \mathrm{OH}\right.$, $99.5 \%, A R$ ), and petroleum ether (AR) were purchased from Sinopharm Chemical Reagent Co., Ltd.

Synthesis of $\mathbf{A u}_{60} \mathbf{S}_{6}\left(\mathbf{S C H}_{\mathbf{2}} \mathbf{P h}\right)_{\mathbf{3 6}}$ nanoclusters. All chemicals and reagents were used as received. Ten milligrams of $\mathrm{Au}_{38}\left(\mathrm{SC}_{2} \mathrm{H}_{4} \mathrm{Ph}\right)_{24}$ nanoclusters was dissolved in $1 \mathrm{ml}$ of toluene containing $0.5 \mathrm{ml}$ of $\mathrm{PhCH}_{2} \mathrm{SH}$. Next, the reaction proceeded overnight at $100{ }^{\circ} \mathrm{C}$ under nitrogen atmosphere, and then was terminated by the addition of excess methanol. The crude product was washed with petroleum ether and methanol four times, dissolved in dichloromethane (DCM), and then subjected to separation and purification by PTLC. Single crystals of the purified nanoclusters were grown by the vapour diffusion of acetonitrile into a toluene solution of the purified nanoclusters at $5{ }^{\circ} \mathrm{C}$, and black-coloured crystals formed after one week. $\mathrm{Au}_{38}\left(\mathrm{SC}_{2} \mathrm{H}_{4} \mathrm{Ph}\right)_{24}$ were prepared according to previous reports ${ }^{44}$.

Characterization. Ultraviolet-visible-near-infrared absorption measurements were performed on a Shimadzu UV-3600 spectrophotometer (DCM as solvent). The single crystal diffraction data of $\mathrm{Au}_{60} \mathrm{~S}_{6}\left(\mathrm{SCH}_{2} \mathrm{Ph}\right)_{36}$ was recorded on a Bruker APEXDUO X-ray Diffractometer (Bruker, Germany). ESI-MS was conducted on a Waters Q-TOF mass spectrometer equipped with a Z-spray source, and the source temperature was kept at $70^{\circ} \mathrm{C}$. To prepare the samples for ESI-MS analysis, $\mathrm{Au}_{60} \mathrm{~S}_{6}\left(\mathrm{SCH}_{2} \mathrm{Ph}\right)_{36}$ was dissolved in toluene $\left(\sim 0.5 \mathrm{mg} \mathrm{ml}^{-1}\right)$ and then diluted $(1 / 1, v / v)$ with an ethanol solution containing $0.5 \mathrm{mM}$ CsOAc. The sample was directly infused into the chamber at $5 \mu \mathrm{min}^{-1}$. The spray voltage was $2.20 \mathrm{kV}$, and the cone voltage was kept at $60 \mathrm{~V}$. The solution photoluminescence spectra of $\mathrm{Au}_{60} \mathrm{~S}_{6}\left(\mathrm{SCH}_{2} \mathrm{Ph}\right)_{36}$ nanoclusters were recorded on a Fluorolog-3-21, Tempro-01 spectrofluorometer (HORIBA Jobin Yvon), and the excitation wavelength was kept at $514 \mathrm{~nm}$ with a slit of $10 \mathrm{~nm}\left(\mathrm{OD}_{514} \sim 0.047\right.$, measured by Ultraviolet-visiblenear-infrared spectrophotometer). The solid photoluminescence spectra of $\mathrm{Au}_{60} \mathrm{~S}_{6}\left(\mathrm{SCH}_{2} \mathrm{Ph}\right)_{36}$ nanoclusters were recorded on a laser confocal scanning Raman/fluorescence scope (HORIBA Jobin Yvon) and laser $(514 \mathrm{~nm})$ power is $0.5 \mathrm{~mW}$. The PTLC plates were eluted with DCM/petroleum ether mixture $(1 / 1$, $\mathrm{v} / \mathrm{v})$ at room temperature under air atmosphere.

Data availability. The X-ray crystallographic coordinates for structures reported in this article (see Supplementary Table 1 and Supplementary Data 1) have been deposited at the Cambridge Crystallographic Data Centre (CCDC), under deposition number CCDC 1526120 . The data can be obtained free of charge from the Cambridge Crystallographic Data Centre via www.ccdc.cam.ac.uk/data_request/cif. All other data are available from the authors on reasonable request.

\section{References}

1. Ehlich, H., Schier, A. \& Schmidbaur, H. Aurophilicity-based one-dimensional arrays of gold(I) phenylene-1,3-and-1,4-dithiolates. Inorg. Chem. 41, 3721-3727 (2002).

2. Vericat, C., Vela, M. E., Benitez, G., Carro, P. \& Salvarezza, R. C. Self-assembled monolayers of thiols and dithiols on gold: new challenges for a well-known system. Chem. Soc. Rev. 39, 1805-1834 (2010).

3. Häkkinen, H. The gold-sulfur interface at the nanoscale. Nat. Chem. 4, 443-445 (2012).

4. Kim, F., Connor, S., Song, H., Kuykendall, T. \& Yang, P. Platonic gold nanocrystals. Angew. Chem. Int. Ed. Engl. 43, 3673-3677 (2004).

5. Xie, J., Lee, J. Y. \& Wang, D. I. C. Seedless, surfactantless, high-yield synthesis of branched gold nanocrystals in HEPES buffer solution. Chem. Mater. 19, 2823-2830 (2007).

6. Zeng, J., Zhang, Q., Chen, J. \& Xia, Y. A comparison study of the catalytic properties of Au-based nanocages, nanoboxes, and nanoparticles. Nano Lett. 10, 30-35 (2010).

7. Chen, H. et al. Understanding the photothermal conversion efficiency of gold nanocrystals. Small 6, 2272-2280 (2010).

8. Angelome, P. C. et al. Seedless synthesis of single crystalline Au nanoparticles with unusual shapes and tunable LSPR in the near-IR. Chem. Mater. 24, 1393-1399 (2012). 
9. Chen, S. et al. Gold nanoelectrodes of varied size: transition to molecule-like charging. Science 280, 2098-2101 (1998).

10. Shibu, E. S., Habeeb Muhammed, M. A., Tsukuda, T. \& Pradeep, T. Ligand exchange of $\mathrm{Au}_{25} \mathrm{SG}_{18}$ leading to functionalized gold clusters: spectroscopy, kinetics, and luminescence. J. Phys. Chem. C 112, 12168-12176 (2008).

11. Gruene, P. et al. Structures of neutral $\mathrm{Au}_{7}, \mathrm{Au}_{19}$, and $\mathrm{Au}_{20}$ clusters in the gas phase. Science 321, 674-676 (2008).

12. Dolamic, I., Knoppe, S., Dass, A. \& Bürgi, T. First enantioseparation and circular dichroism spectra of $\mathrm{Au}_{38}$ clusters protected by achiral ligands. Nat. Commun. 3, 798 (2012)

13. Kurashige, W., Yamazoe, S., Kanehira, K., Tsukuda, T. \& Negishi, Y. Selenolateprotected $\mathrm{Au}_{38}$ nanoclusters: isolation and structural characterization. J. Phys. Chem. Lett. 4, 3181-3185 (2013).

14. Zhang, X.-D. et al. Ultrasmall $\mathrm{Au}_{10-12}(\mathrm{SG})_{10-12}$ nanomolecules for high tumor specificity and cancer radiotherapy. Adv. Mater. 26, 4565-4568 (2014).

15. Gan, Z. et al. Fluorescent gold nanoclusters with interlocked staples and a fully thiolate-bound kernel. Angew. Chem. Int. Ed. Engl. 55, 11567-11571 (2016).

16. Heaven, M. W., Dass, A., White, P. S., Holt, K. M. \& Murray, R. W. Crystal structure of the gold nanoparticle $\left[\mathrm{N}\left(\mathrm{C}_{8} \mathrm{H}_{17}\right)_{4}\right]\left[\mathrm{Au}_{25}\left(\mathrm{SCH}_{2} \mathrm{CH}_{2} \mathrm{Ph}\right)_{18}\right]$. J. Am. Chem. Soc. 130, 3754-3755 (2008).

17. Qian, H., Eckenhoff, W. T., Zhu, Y., Pintauer, T. \& Jin, R. Total structure determination of thiolate-protected $\mathrm{Au}_{38}$ nanoparticles. J. Am. Chem. Soc. 132, 8280-8281 (2010)

18. Song, Y. et al. Crystal structure of selenolate-protected $\mathrm{Au}_{24}(\mathrm{SeR})_{20}$ nanocluster. J. Am. Chem. Soc. 136, 2963-2965 (2014).

19. Tian, S. et al. Structural isomerism in gold nanoparticles revealed by X-ray crystallography. Nat. Commun. 6, 8667 (2015).

20. Liao, L. et al. Structure of chiral $\mathrm{Au}_{44}(2,4-\mathrm{DMBT})_{26}$ nanocluster with an 18-electron shell closure. J. Am. Chem. Soc. 138, 10425-10428 (2016).

21. Zeng, C., Chen, Y., Kirschbaum, K. J., Lambright, K. J. \& Jin, R. Emergence of hierarchical structural complexities in nanoparticles and their assembly. Science 354, 1580-1584 (2016).

22. Tsunoyama, R., Tsunoyama, H., Pannopard, P., Limtrakul, J. \& Tsukuda, T. MALDI mass analysis of $11 \mathrm{kDa}$ gold clusters protected by octadecanethiolate Ligands. J. Phys. Chem. C 114, 16004-16009 (2010).

23. Zeng, C., Chen, Y., Li, G. \& Jin, R. Magic size $\mathrm{Au}_{64}\left(\mathrm{~S}_{-} \mathrm{c}^{-} \mathrm{C}_{6} \mathrm{H}_{11}\right)_{32}$ nanocluster protected by cyclohexanethiolate. Chem. Mater. 26, 2635-2641 (2014).

24. Nimmala, P. R., Yoon, B., Whetten, R. L., Landman, U. \& Dass, A. $\operatorname{Au}_{67}(\mathrm{SR})_{35}$ nanomolecules: characteristic size-specific optical, electrochemical, structural properties and first-principles theoretical analysis. J. Phys. Chem. A 117, 504-517 (2013).

25. Negishi, Y., Sakamoto, C., Ohyama, T. \& Tsukuda, T. Synthesis and the origin of the stability of thiolate-protected $\mathrm{Au}_{130}$ and $\mathrm{Au}_{187}$ clusters. J. Phys. Chem. Lett. 3, 1624-1628 (2012).

26. Qian, H. \& Jin, R. Ambient synthesis of $\mathrm{Au}_{144}(\mathrm{SR})_{60}$ nanoclusters in methanol. Chem. Mater. 23, 2209-2217 (2011).

27. Zeng, C., Liu, C., Chen, Y., Rosi, N. L. \& Jin, R. Gold-thiolate ring as a protecting motif in the $\mathrm{Au}_{20}(\mathrm{SR})_{16}$ nanocluster and implications. J. Am. Chem. Soc. 136, 11922-11925 (2014).

28. Crasto, D., Malola, S., Brosofsky, G., Dass, A. \& Häkkinen, H. Single crystal $\mathrm{XRD}$ structure and theoretical analysis of the chiral $\mathrm{Au}_{30} \mathrm{~S}(\mathrm{~S}-t-\mathrm{Bu})_{18}$ cluster. J. Am. Chem. Soc. 136, 5000-5005 (2014).

29. Liu, C. et al. Observation of body-centered cubic gold nanocluster. Angew. Chem. Int. Ed. Engl. 54, 9826-9829 (2015).

30. Bino, A., Johnston, D. C. \& Stiefel, E. I. Tetranuclear sulfido-bridged complex of Cr(III) having a strongly magnetic ground state. US Patent 4,832,877 (1989).

31. Parish, R. V., Salehi, Z. \& Pritchard, R. G. Five-coordinate sulfur in a polymeric copper(I) thiolate complex. Angew. Chem. Int. Ed. Engl. 36, 251-253 (1997).

32. Fan, Z. \& Zhang, H. Crystal phase-controlled synthesis, properties and applications of noble metal nanomaterials. Chem. Soc. Rev. 45, 63-82 (2016).

33. Chen, M. et al. Synthesis and self-assembly of fcc phase FePt nanorods. J. Am. Chem. Soc. 129, 6348-6349 (2007).

34. Duan, H. et al. Ultrathin rhodium nanosheets. Nat. Commun. 5, 3093 (2014).

35. Huang, X. et al. Synthesis of hexagonal close-packed gold nanostructures. Nat. Commun. 2, 292 (2011).

36. Yang, S., Peng, Z. \& Yang, H. Platinum lead nanostructures: formation, phase behavior, and electrocatalytic properties. Adv. Funct. Mater. 18, 2745-2753 (2008).
37. Liao, H., Zhu, J. \& Hou, Y. Synthesis and electrocatalytic properties of PtBi nanoplatelets and PdBi nanowires. Nanoscale 6, 1049-1055 (2014).

38. Novgorodova, M. I., Gorshkov, A. I. \& Mokhov, A. V. Native silver and its new structural modifications. Zap. Vses. Mineral. Obschch. 108, 552-563 (1979).

39. Taneja, P., Banerjee, R. \& Ayyub, P. Observation of a hexagonal $4 \mathrm{H}$ phase in nanocrystalline silver. Phys. Rev. B 64, 033405 (2001).

40. Liu, X., Luo, J. \& Zhu, J. Size effect on the crystal structure of silver nanowires. Nano Lett. 6, 408-412 (2006).

41. Fan, Z. et al. Stabilization of $4 \mathrm{H}$ hexagonal phase in gold Nanoribbons. Nat. Commun. 6, 7684 (2015).

42. Higaki, T. et al. Controlling the atomic structure of $\mathrm{Au}_{30}$ nanoclusters by a ligand-based strategy. Angew. Chem. Int. Ed. Engl. 55, 6694-6697 (2016).

43. Liao, L. et al. Transition-sized $\mathrm{Au}_{92}$ nanoparticle bridging non-fcc-structured gold nanoclusters and fcc-structured gold nanocrystals. Chem. Commun. 52, 12036-12039 (2016).

44. Qian, H., Zhu, Y. \& Jin, R. Size-focusing synthesis, optical and electrochemical properties of monodisperse $\mathrm{Au}_{38}\left(\mathrm{SC}_{2} \mathrm{H}_{4} \mathrm{Ph}\right)_{24}$ nanoclusters. ACS Nano 3, 3795-3803 (2009).

\section{Acknowledgements}

We would like to thank National Natural Science Foundation of China (Nos 21222301, 51502299, 21528303, 21171170, 21501182), Postdoctoral Science Foundation of China (No 2015M571951), National Basic Research Program of China (No 2013CB934302), the Ministry of Human Resources and Social Security of China, Anhui Provincial Natural Science Foundation (1608085QB31), the Innovative Program of Development Foundation of Hefei Centre for Physical Science and Technology (2014FXCX002), Hefei Science Center, CAS (user of potential: 2015HSC-UP003), the CAS/SAFEA International Partnership Program for Creative Research Teams, and the Hundred Talents Program of the Chinese Academy of Sciences for financial support.

\section{Author contributions}

Z.G. conceived and carried out the experiment and UV/Vis/NIR characterization of $\mathrm{Au}_{60} \mathrm{~S}_{6}\left(\mathrm{SCH}_{2} \mathrm{Ph}\right)_{36}$ with the assistance of C.Y. C.W. assisted the photoluminescence data collection. J.C. analysed the single crystal structure of $\mathrm{Au}_{60} \mathrm{~S}_{6}\left(\mathrm{SCH}_{2} \mathrm{Ph}\right)_{36}$. J.W. and A.X. assisted the crystallization of $\mathrm{Au}_{60} \mathrm{~S}_{6}\left(\mathrm{SCH}_{2} \mathrm{Ph}\right)_{36}$. M.-B.L. and S.Z. assisted the ESI-MS analysis. L.L. collected the single crystal data. Z.W. designed the study, supervised the project and analysed the data. All authors contributed to the preparation of the manuscript.

\section{Additional information}

Supplementary Information accompanies this paper at http://www.nature.com/ naturecommunications

Competing interests: The authors declare no competing financial interests.

Reprints and permission information is available online at http://npg.nature.com/ reprintsandpermissions/

How to cite this article: Gan, Z. et al. The fourth crystallographic closest packing unveiled in the gold nanocluster crystal. Nat. Commun. 8, 14739 doi: $10.1038 /$ ncomms14739 (2017).

Publisher's note: Springer Nature remains neutral with regard to jurisdictional claims in published maps and institutional affiliations.

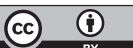

This work is licensed under a Creative Commons Attribution 4.0 International License. The images or other third party material in this article are included in the article's Creative Commons license, unless indicated otherwise in the credit line; if the material is not included under the Creative Commons license, users will need to obtain permission from the license holder to reproduce the material. To view a copy of this license, visit http://creativecommons.org/licenses/by/4.0/

(C) The Author(s) 2017 